\title{
AQP2 is regulated by estradiol in human endometrium and is associated with spheroid attachment in vitro
}

\author{
RONGHUAN HE ${ }^{1 *}$, WENLUN HAN ${ }^{2 *}$, YANJUN HU $^{1}$, XIJING CHEN $^{1}$, XIAOLING HU ${ }^{1}$ and YIMIN ZHU ${ }^{1}$ \\ ${ }^{1}$ Department of Reproductive Endocrinology, Women's Hospital, School of Medicine, Zhejiang University, Hangzhou, \\ Zhejiang 310006; ${ }^{2}$ Department of Nephrology, Tongde Hospital of Zhejiang, Hangzhou, Zhejiang 310012, P.R. China
}

Received October 5, 2017; Accepted June 14, 2018

DOI: $10.3892 / \mathrm{mmr} .2019 .10338$

\begin{abstract}
E2) and aquaporin 2 (AQP2) are associated with endometrial receptivity, and E2 directly regulates AQP2 expression in endometrial cancer cells. The present study aimed to investigate the role of AQP2 in embryo implantation. Normal endometrial samples were collected at the Women's Hospital (Hangzhou, China) from women seeking in vitro fertilization and embryo transfer; women with endometrial abnormalities were excluded from the study. Samples were categorized into early-mid proliferative, late proliferative, early secretory, mid-secretory and late secretory phase groups, according to the menstrual cycle. The mRNA and protein expression levels of AQP2 were assessed in normal human endometrium in response to $\mathrm{E} 2$ via reverse transcription-quantitative polymerase chain reaction and western blotting, respectively. The effects of AQP2 on spheroid attachment were assessed using an in vitro co-culture assay with small interfering (si)RNA against AQP2. The highest expression levels of AQP2 were observed in the late proliferative and mid-secretory phases, with the lowest levels detected in the early proliferative and late secretory phases. In addition, treatment with $10^{-9}$ or $10^{-7} \mathrm{M} \mathrm{E} 2$ for $24 \mathrm{~h}$ upregulated AQP2 in the cultured endometrium. Knockdown of AQP2 by siRNA significantly decreased JAr spheroid attachment; however, this effect was significantly reversed when AQP2 siRNA-transfected cells were treated with $10^{-7} \mathrm{M}$ E2. The results of the present study suggested that AQP2 expression
\end{abstract}

Correspondence to: Dr Yimin Zhu, Department of Reproductive Endocrinology, Women's Hospital, School of Medicine, Zhejiang University, 1 Xueshi Road, Hangzhou, Zhejiang 310006, P.R. China E-mail: zhuyim@zju.edu.cn

Abbreviations: $\mathrm{AQP} 2$, aquaporin 2; Cq, quantification cycle; E2, 17ß-estradiol; ER, estrogen receptor; ER-ERE, estrogen receptor-estrogen response element; IVF, in vitro fertilization; RT-qPCR, reverse transcription-quantitative polymerase chain reaction

*Contributed equally

Key words: 17ß-estradiol, aquaporin 2, endometrium, human, implantation levels in human endometrium may be mediated by estrogen, and low AQP2 expression levels may be a potential cause of impaired uterine receptivity.

\section{Introduction}

In vitro fertilization (IVF) is a first-line treatment for female infertility. It involves egg collection, fertilization of the eggs with sperm in the laboratory, followed by the transfer of one or more viable embryos to the uterus, with the hope of successful pregnancy. In 2012, an estimated 5 million children were born from IVF (1). During IVF, it is necessary to collect and fertilize numerous eggs in order to increase the number of high-quality embryos available for transfer, and multi-follicular development is induced by hormonal stimulation. In women without ovarian function, $17 \beta$-estradiol (E2) and progesterone are the two hormones that need to be provided to achieve a receptive endometrial environment (2). Clinical evidence has suggested that endometrial receptivity during controlled ovarian hyperstimulation is reduced, which may increase the circulating levels of E2 to supraphysiological levels $(3,4)$. Supraphysiological serum levels of E2 may exert a negative effect on endometrial receptivity, but without affecting embryo quality (5-7). Using a step-down protocol to decrease the plasma levels of E2 prior to embryo transfer may improve the implantation rate (8). Nevertheless, the mechanisms underlying the effects of supraphysiological levels of E2 on endometrial receptivity remain unclear. Proteomic studies have revealed the differences between receptive and non-receptive endometria during the implantation window (9); however, the effect of E2 is still poorly understood (8).

The roles of aquaporins (AQPs) in the reproductive system have previously been examined (10-12). AQPs serve functional and distinctive collaborative roles in regulating the amount of water in the mammalian uterus and oviduct $(13,14)$. It has previously been demonstrated that in the human endometrium, AQP2 is expressed in a menstruation-dependent manner, suggesting that AQP2 may serve physiological roles in uterine receptivity (15). In addition, AQP2 expression in human endometrium is positively correlated with serum E2 levels, suggesting that E2 may regulate AQP2 expression $(15,16)$. A previous study strongly suggested that AQP2 may be regulated by $\mathrm{E} 2$ via an estrogen receptor-estrogen response element (ER-ERE) in endometrial carcinoma cells (17). However, 
whether E2 regulates AQP2 expression in normal human endometrium has yet to be determined. In addition, the role of AQP2 in embryo implantation is largely unknown. Therefore, the present study aimed to investigate whether E2 regulates AQP2 expression in normal human endometrial tissues, and to determine the role of AQP2 in embryo implantation.

\section{Patients and methods}

Patients and sample collection. Normal endometrial samples were collected from women seeking IVF and embryo transfer $(n=113$; median age, 32 years; age range, 25-38 years) at the Women's Hospital (Hangzhou, China). Women with endometrial abnormalities were excluded from this study. Endometrial samples were collected from women undergoing hysterectomies between August 2015 and February 2016 on days 6-15 (proliferative phase, $\mathrm{n}=38$ ) or 16-28 (secretory phase, $\mathrm{n}=75$ ) of the menstrual cycle. None of the women were treated with cycle-altering medication. Shortly after collection, the endometrial samples were cut with a scalpel into pieces (size, $1.0 \times 0.5 \mathrm{~cm}$ ), and either snap frozen in liquid nitrogen and stored at $-70^{\circ} \mathrm{C}$ for the extraction of protein and mRNA, or fixed with $4 \%$ paraformaldehyde for $24 \mathrm{~h}$ at $4^{\circ} \mathrm{C}$ prior to pathological examination. According to Noyes pathological diagnostic criteria (18), endometrial samples were categorized as early-mid proliferative (days 5-10), late proliferative (days 11-14), early secretory (days 15-18), mid-secretory (days 19-24) and late secretory (days 25-28) phase groups following hematoxylin and eosin staining. Briefly, the sections were prepared at room temperature and were stained with hematoxylin $(0.5 \%)$ and eosin $(0.5 \%)$ for $25-28 \mathrm{~min}$ at room temperature, after which, they were examined using a light microscope (Olympus Corporation, Tokyo, Japan).

The present study was approved by the Ethics Committee of the School of Medicine of Zhejiang University (Hangzhou, China). Written informed consent was obtained from each patient prior to tissue collection and enrolment in the present study.

Tissue culture. Endometrial explant culture was performed as previously described (19). Briefly, endometrial explants in the proliferative phase from hysterectomy specimens were minced using a scalpel $(2-3 \mathrm{~mm})$, placed in a 12-well dish with the luminal epithelial surface facing upward, and cultured in RPMI-1640 containing 10\% charcoal-stripped fetal bovine serum (FBS; Hyclone; GE Healthcare Life Sciences, Logan, UT, USA) at $37^{\circ} \mathrm{C}$ in a humidified environment containing $5 \% \mathrm{CO}_{2}$. After $24 \mathrm{~h}$, the tissues ( $\mathrm{n}=5)$ were exposed to ethanol (control; same volume as in the E2 groups) or various concentrations of E2 $\left(10^{-9}\right.$ to $10^{-5} \mathrm{M}$; cat. no. E2257; Sigma-Aldrich Merck KGaA, Darmstadt, Germany) for an additional $24 \mathrm{~h}$ at $4^{\circ} \mathrm{C}$. Rat kidney tissues were collected from a 4-week old male Sprague-Dawley rat (weight, $200 \mathrm{~g}$; Shanghai SLAC Laboratory Animal Co., Ltd., Shanghai, China) and were used as positive controls for AQP expression, as previously described (20). The rat was routinely maintained and received no prior treatment; kidney tissues were cultured in the same manner as endometrial tissues.

Reverse transcription-quantitative polymerase chain reaction $(R T-q P C R)$. Following treatment, endometrial tissues were either snap frozen in liquid nitrogen and stored at $-70^{\circ} \mathrm{C}$ for the extraction of RNA and protein, or processed to obtain $4-\mu \mathrm{m}$ sections. For RNA extraction, endometrial tissues (50-100 mg) were homogenized in $1 \mathrm{ml}$ RNAiso Plus (Sangon Biotech Co., Ltd., Shanghai, China). The supernatant was collected, mixed with $200 \mu \mathrm{l}$ trichloromethane and agitated for $30 \mathrm{sec}$. After centrifugation at $240 \mathrm{x} \mathrm{g}$ for $15 \mathrm{~min}$ at $4^{\circ} \mathrm{C}$, the supernatant was collected and mixed with an equal volume of isopropyl alcohol. The mixture was centrifuged at $240 \mathrm{x}$ g for $10 \mathrm{~min}$ at $4{ }^{\circ} \mathrm{C}$, and the precipitate was dissolved in $75 \%$ ethanol. Total RNA was extracted after being centrifuged at $120 \mathrm{x} \mathrm{g}$ for $5 \mathrm{~min}$ at $4^{\circ} \mathrm{C}$, and was dissolved in DEPC water. Total RNA was processed using DNase to remove DNA contamination. Subsequently, cDNA was synthesized using the Reverse Transcriptase kit (Takara Bio, Inc., Otsu, Japan); the RNA was heated to $37^{\circ} \mathrm{C}$ for $60 \mathrm{~min}$, followed by incubation at $95^{\circ} \mathrm{C}$ for $5 \mathrm{~min}$. Eventually, the cDNA was stored at $-20^{\circ} \mathrm{C}$ for further analysis. RT-qPCR was conducted on an Icycler ${ }^{\mathrm{TM}}$ Optical Module using the $\mathrm{iQ}^{\mathrm{TM}} \mathrm{SYBR}^{\circledR}$ Green Supermix (both Bio-Rad Laboratories, Inc., Hercules, CA, USA). The primers used were as follows: AQP2 forward, 5'-TGGGCCATATGT GCTATGGAGA-3' and reverse, 5'-AAGGACACTCAG GTGCCAGGA-3' (amplicon size, 142bp); and as an internal reference, GAPDH forward, 5'-CAGGGCTGCTTTTAA CTCTGG-3' and reverse, 5'-TGGGTGGAATCATATTGGA ACA-3' (amplicon size, 102bp). For qPCR, the following thermocycling program was adopted: Initial denaturation at $94^{\circ} \mathrm{C}$ for $5 \mathrm{~min} ; 30$ cycles of denaturation at $94^{\circ} \mathrm{C}$ for $30 \mathrm{sec}$, annealing at $65^{\circ} \mathrm{C}$ for $30 \mathrm{sec}$ and extension at $72^{\circ} \mathrm{C}$ for $30 \mathrm{sec}$, and a final extension step at $72^{\circ} \mathrm{C}$ for $10 \mathrm{~min}$. Data were analyzed using the comparative quantification cycle $(\mathrm{Cq})$ method $(21,22)$ : Relative expression $=2^{-\Delta \Delta \mathrm{Cq}}$, where $\Delta \Delta \mathrm{Cq}=\Delta \mathrm{Cq}$ (treated group) $-\Delta \mathrm{Cq}$ (control group), and $\Delta \mathrm{Cq}=\mathrm{Cq}$ (sample)-Cq (internal control).

Immunofluorescence. Endometrial samples (1.0x0.5 cm) were fixed as aforementioned, sectioned at $4 \mu \mathrm{m}$, permeabilized with Triton-X, blocked with $1 \%$ bovine serum albumin (Hyclone; GE Healthcare Life Sciences) at $4^{\circ} \mathrm{C}$ for $1 \mathrm{~h}$ and incubated with goat anti-AQP2 primary antibody (1:100; cat. no. sc-9882; Santa Cruz Biotechnology, Inc., Dallas, TX, USA) at $4^{\circ} \mathrm{C}$ overnight. The samples were subsequently incubated with Alexa Fluor ${ }^{\circledR} 488$ rabbit anti-goat immunoglobulin G (IgG) secondary antibodies (1:200; cat. no. A27012; Invitrogen; Thermo Fisher Scientific, Inc., Waltham, MA, USA) for $2 \mathrm{~h}$ at room temperature. No counterstain was used. The slides were analyzed under a Zeiss LSM 510Meta laser scanning confocal microscope (Carl Zeiss AG, Oberkochen, Germany) or an Olympus BX51TF fluorescence microscope (Olympus Corporation).

Western blot analysis. Western blotting was performed as described previously, without modification (15). Briefly, the proteins were separated by $8 \%$ SDS-PAGE and were transferred onto nitrocellulose membranes. The membranes were blocked in skimmed milk at $4^{\circ} \mathrm{C}$ for $1 \mathrm{~h}$ and were exposed to goat-anti AQP2 (1:500; cat. no. sc-9882; Santa Cruz Biotechnology, Inc.) and goat-anti $\beta$-actin (1:500; cat no. sc-1616; Santa Cruz Biotechnology, Inc.) polyclonal antibodies, respectively, at $4^{\circ} \mathrm{C}$ overnight. Subsequently, membranes were incubated with horseradish peroxidase-labeled rabbit anti-goat IgG 
(1:2,000 for AQP2 and 1:5,000 for $\beta$-actin; cat. no. ZB-2306, OriGene Technologies, Inc., Beijing, China) for $1 \mathrm{~h}$ at room temperature with agitation. Visualization was performed with an enhanced chemiluminescence detection reagent (Santa Cruz Biotechnology, Inc.). The protein bands were analyzed with Quantity One software 3.1 (Bio-Rad Laboratories, Inc.). $\beta$-actin was used as a loading control.

AQP2 silencing by small interfering RNA (siRNA) technology. siRNA sequences against AQP2 were generated using the Silencer siRNA Construction kit (Ambion; Thermo Fisher Scientific, Inc.), according to the manufacturer's protocols. The siRNA sequences against AQP2 were as follows: Forward, 3'-GGUGGGUGGUAAGAGGGAATT-5' and reverse, 3'-UUCCCUCUUACCACCCACCTG-5'. Control scrambled siRNAs were purchased from Ambion (cat. no. AM4611; Thermo Fisher Scientific, Inc.). Ishikawa cells (cat. no. 99040201) were inoculated at 100 cells $/ \mathrm{cm}^{2}$ in minimum essential medium (MEM; both Sigma-Aldrich; Merck KGaA) supplemented with $2 \mathrm{mM}$ glutamine, $1 \%$ non-essential amino acids and 5\% FBS (Hyclone; GE Healthcare Life Sciences) at $37^{\circ} \mathrm{C}$ in an atmosphere containing $5 \% \mathrm{CO}_{2}$. After reaching $70-80 \%$ confluence, the cells were transfected with siRNA duplexes for $24 \mathrm{~h}$ using Lipofectamine ${ }^{\circledR} 2000$ (Invitrogen; Thermo Fisher Scientific, Inc.), according to the manufacturer's protocols. A final concentration of $5 \mathrm{nM}$ AQP2 siRNA was determined to maximally suppress target RNA expression. The same concentration was used for scrambled siRNA transfection.

Evaluation of JAr spheroid attachment onto Ishikawa cells. Attachment of the choriocarcinoma cell line, Jar (HTB-144; American Type Culture Collection, Manassas, VA, USA) to endometrial cells (Ishikawa) was quantified according to an adhesion assay, as previously described (23-25). Briefly, a suspension of $6 \times 10^{5} \mathrm{JAr}$ cells in $6 \mathrm{ml}$ RPMI-1640 medium supplemented with $10 \% \mathrm{FBS}$ was incubated at $37^{\circ} \mathrm{C}$ on a gyratory shaker at $110 \mathrm{rpm}$, in order to generate multicellular spheroids 3 days following culture initiation. Ishikawa cells were inoculated at 100 cells $/ \mathrm{cm}^{2}$ in 12 -well plates containing MEM supplemented with $2 \mathrm{mM}$ glutamine, $1 \%$ non-essential amino acids and 5\% FBS (Hyclone; GE Healthcare Life Sciences) at $37^{\circ} \mathrm{C}$ in an atmosphere containing $5 \% \mathrm{CO}_{2}$. At $40 \%$ confluence, Ishikawa cells were transfected with scrambled siRNA or siRNA $(6 \mathrm{nM})$ duplexes targeting AQP2 for $24 \mathrm{~h}$, as mentioned, and were then treated with or without E2 $\left(10^{-7} \mathrm{M}\right)$ for $24 \mathrm{~h}$ at $37^{\circ} \mathrm{C}$ in an atmosphere containing $5 \% \mathrm{CO}_{2}$. Subsequently, spheroids $(60-200 \mu \mathrm{m})$ were transferred onto a confluent monolayer of Ishikawa cells. The cultures were maintained in culture medium (RPMI-1640 medium with 10\% FBS) for $1 \mathrm{~h}$ at $37^{\circ} \mathrm{C}$ in a $5 \% \mathrm{CO}_{2}$ incubator. After $1 \mathrm{~h}$, spheroid adhesion to the endometrial monolayer was quantified by centrifugation of the 12 -well plates with the cell-spheroid surface facing down at $10 \mathrm{x}$ g for $10 \mathrm{~min}$ at $4^{\circ} \mathrm{C}$. The unattached spheroids were removed by centrifugation. Attached spheroids were visualized under an anatomical microscope (magnification, $\mathrm{x} 100$ ) and were counted under a phase contrast microscope (magnification, $\mathrm{x} 400$ ). Results were expressed as a percentage of the total number of spheroids (attachment rate $(\%)=$ number of attached spheroids/total spheroids) x 100 .

Statistical analysis. All experiments were performed three times. All data were normally distributed and expressed as the means \pm standard error of the mean. One-way analysis of variance followed by a Tukey's post hoc test was used to assess differences among groups. SPSS 11.0 for Windows (SPSS, Inc., Chicago, IL, USA) was used for statistical analysis. $\mathrm{P}<0.05$ was considered to indicate a statistically significant difference.

\section{Results}

Menstrual cycle-dependent expression of AQP2 in human endometrium. AQP2 mRNA and protein were expressed in human endometrium, in a menstrual cycle-dependent manner (Fig. 1A). The lowest levels of AQP2 expression were observed in the early-mid proliferative and late secretory phases (Fig. 1A and B). The highest AQP2 expression levels were obtained in the late proliferative and mid-secretory phases (Fig. 1A and B). E2 levels are altered throughout the menstrual cycle (with a peak at day 12-13) (26) and these results suggested that AQP2 expression in the human endometrium may be E2-dependent.

Treatment with $10^{-7} \mathrm{M}$ E2 increases AQP2 expression in cultured human endometrium. Immunofluorescence revealed that AQP2 was mainly located in the glandular and luminal epithelia (Fig. 2Aa-d), in accordance with our previous report (15). Stronger immunofluorescence signals for AQP2 were observed in the $10^{-7} \mathrm{M}$ E2 group (Fig. 2Ac); however, treatment with $10^{-5} \mathrm{M}$ E2 notably decreased the fluorescence of AQP2 (Fig. 2Ad) compared with $10^{-7} \mathrm{M} \mathrm{E2}$ (Fig. 2Ac). Western blotting (Fig. 2B) and RT-qPCR (Fig. 2C) analyses revealed that endometrial tissues in the $10^{-9}$ and $10^{-7} \mathrm{M}$ E2-treated groups exhibited significantly higher expression levels of AQP2 compared with in the control group. AQP2 expression levels were significantly reduced in response to treatment with $10^{-5} \mathrm{M}$ E2 compared within response to $10^{-7} \mathrm{M}$ E2 (Fig. 2B and C). Therefore, $10^{-7} \mathrm{M}$ E2 was selected for subsequent experimentation to assess the effects of E2 on the endometrium.

Effects of AQP2 RNAi on Ishikawa cells. To confirm the silencing efficiency of AQP2 siRNA by RT-qPCR and western blotting, Ishikawa cells were harvested $24 \mathrm{~h}$ post-transfection with scrambled or AQP2 siRNA duplexes. Compared with in the scrambled siRNA group, Ishikawa cells transfected with AQP2 siRNA exhibited significant reductions in the mRNA and protein expression levels of AQP $2[\sim 81.0 \pm 2.1 \%(\mathrm{P}<0.05)$ and $\sim 72.0 \pm 1.9 \%(\mathrm{P}<0.05)$, respectively] (Fig. 3).

AQP2 siRNA reduces spheroid attachment. JAr spheroids at a diameter of 60-200 $\mu \mathrm{m}$ were used to assess JAr cell attachment onto endometrial Ishikawa cells (Fig. 4A). As presented in Fig. 4B, knockdown of AQP2 by siRNA resulted in a decreased attachment rate in the in vitro attachment model. Attachment rates were: $80.6 \pm 2.2,62.4 \pm 1.5,82.1 \pm 2.2$ and $85.4 \pm 2.4 \%$ in the scrambled siRNA (control), AQP2 siRNA, $10^{-7} \mathrm{M}$ E2 + AQP2 
A

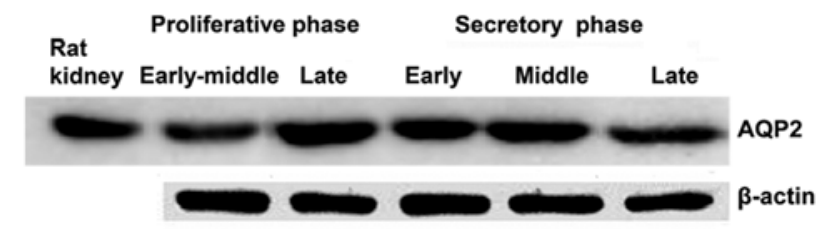

B

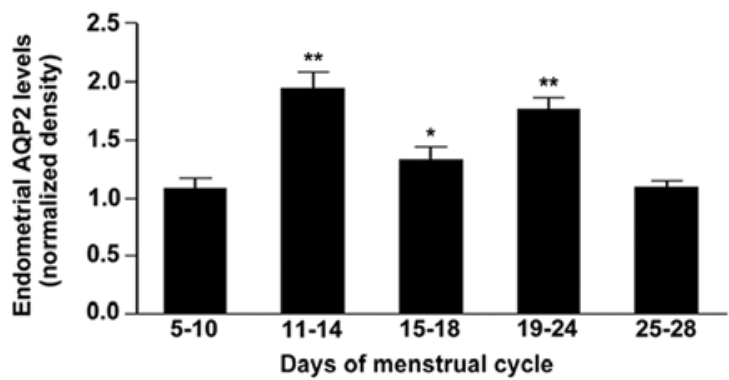

Figure 1. Western blot analysis of AQP2 protein expression in human endometrium at different phases of the menstrual cycle. (A) A 29kDa band, which represented AQP2 protein, was detected in the rat kidney (positive control) and human endometrium. (B) Normalized density of the bands was determined using $\beta$-actin as an internal reference. Data are presented as the means \pm standard error of the mean. "P<0.05, ${ }^{* *} \mathrm{P}<0.01$ vs. the early-mid proliferative phase of the menstrual cycle (5-10 days). AQP2, aquaporin 2.
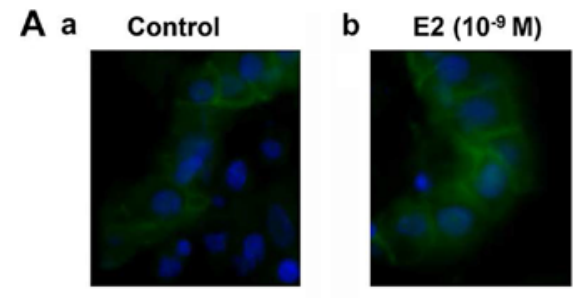

B

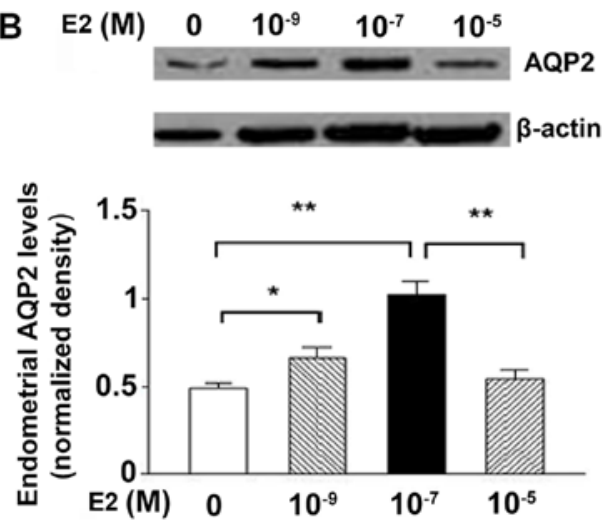

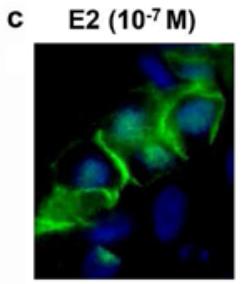

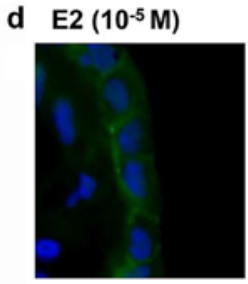

C

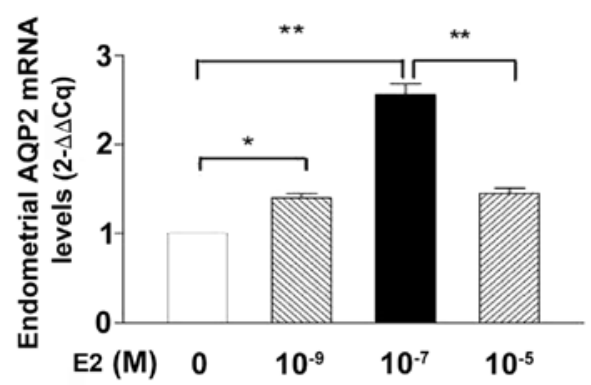

Figure 2. Effects of E2 on AQP2 expression in cultured normal human endometrium. (A) AQP2 stained with Alexa Fluor ${ }^{\circledR} 488$ (green) was mainly located in the glandular and luminal epithelia in normal human endometrial tissues. Magnification, $\mathrm{x} 400$. (a) Control group, (b) $10^{-9} \mathrm{M} \mathrm{E} 2$ group, (c) $10^{-7} \mathrm{M}$ E2 group, (d) $10^{-5} \mathrm{M}$ E2 group. E2 $\left(10^{-9}\right.$ and $\left.10^{-7} \mathrm{M}\right)$ increased AQP2 (B) protein and (C) mRNA expression levels. AQP2 expression levels were significantly reduced in response to treatment with $10^{-5} \mathrm{M}$ E2 compared with in response to $10^{-7} \mathrm{M} \mathrm{E} 2$. Data are presented as the means \pm standard error of the mean of triplicate experiments. At least three independent experiments were performed for each measurement. ${ }^{*} \mathrm{P}<0.05,{ }^{* *} \mathrm{P}<0.01$. AQP2, aquaporin 2 ; E2, $17 \beta$-estradiol.

siRNA, and $10^{-7} \mathrm{M}$ E2 groups, respectively. There were no differences between the $10^{-7} \mathrm{E} 2$ and $10^{-7} \mathrm{E} 2+$ siRNA groups, between the $10^{-7} \mathrm{E} 2$ and control groups, and between the $10^{-7}$ $\mathrm{E} 2+$ siRNA groups and control group (all $\mathrm{P}>0.05$ ); however, a significant difference was detected between the siRNA and $10^{-7} \mathrm{E} 2$ groups, between the siRNA and $10^{-7} \mathrm{E} 2+$ siRNA groups, and between the siRNA and control groups (all $\mathrm{P}<0.05$ ). These results revealed that knockdown of AQP2 by siRNA significantly decreased JAr spheroid attachment compared with in the control group; however, this effect was significantly reversed when AQP2 siRNA-transfected cells were treated with $10^{-7} \mathrm{M}$ E2 (Fig. 4C). These results indicated that the expression of AQP2 may be associated with spheroid attachment and E2 could improve spheroid attachment since significant differences were observed between the siRNA and control groups, and between the siRNA and $10^{-7} \mathrm{E} 2+$ siRNA groups.

\section{Discussion}

E2 and AQP2 have been associated with endometrial receptivity (5-7,10-12), and E2 can directly regulate AQP2 expression in endometrial cancer cells (15). Therefore, the present study aimed to assess the role of AQP2 in embryo implantation. The results revealed that AQP2 expression in human endometrium maybe mediated by E2. In the present study, abnormally high E2 levels attenuated AQP2 expression, which may be a potential cause of impaired uterine receptivity. These results may be beneficial for investigations regarding women undergoing IVF.

The present study reported temporal alterations in the expression levels of AQP2 throughout the menstrual cycle; AQP2 was observed to be upregulated in human endometrium during the implantation window (mid-secretory phase), supporting the findings of previous studies $(15,16)$. These findings suggested that AQP2 may serve physiological roles 
A

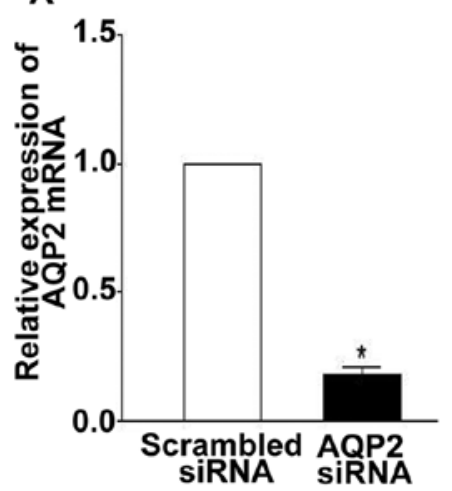

B

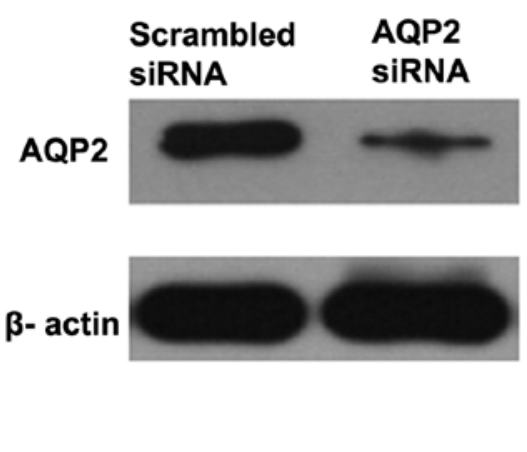

C

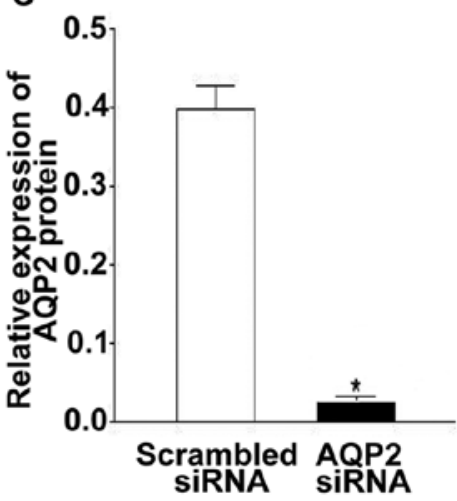

Figure 3. (A) Knockdown of AQP2 in Ishikawa cells by siRNA technology. Ishikawa cells were transfected with siRNA targeting AQP2 or scrambled control siRNA for $24 \mathrm{~h}$. (A) Reverse transcription-quantitative polymerase chain reaction demonstrated that AQP2 mRNA expression levels were decreased by $\sim 80 \%$ in Ishikawa cells. (B and C) Western blotting was used to confirm the inhibition of AQP2 expression mediated by siRNA. Data are presented as the means \pm standard error of the mean. ${ }^{*} \mathrm{P}<0.01$ vs. the scrambled control. AQP2, aquaporin 2 ; siRNA, small interfering RNA.

A

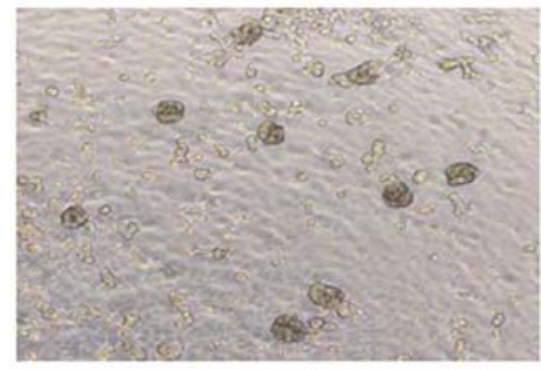

B

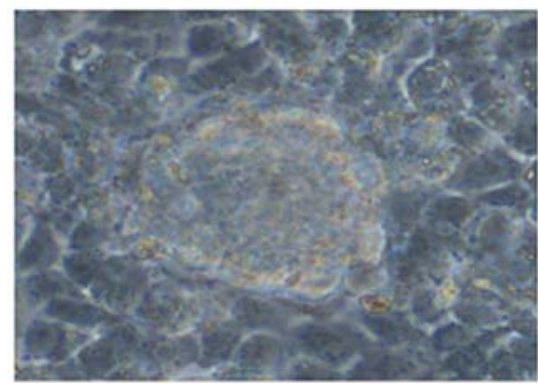

C

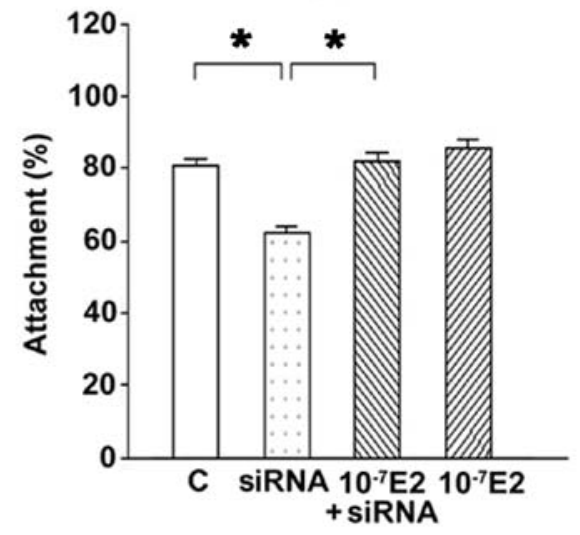

Figure 4. Effects of various treatments on in vitro attachment of JAr spheroids on Ishikawa cells. (A) Spheroids (60-200 mm in diameter) were prepared from JAr cells for co-culture analysis. Spheroids were attached to the Ishikawa monolayer after $1 \mathrm{~h}$ of co-culture as observed under an anatomical microscope (magnification, $\mathrm{x} 100$ ). (B) JAr spheroids were attached to the Ishikawa monolayer as assessed under a phase contrast microscope (magnification, $\mathrm{x} 400$ ). (C) Effects of AQP2 siRNA, $10^{-7} \mathrm{M} \mathrm{E2}+\mathrm{AQP} 2$ siRNA, and $10^{-7} \mathrm{M}$ E2 on the attachment of spheroids on Ishikawa cells. The results were obtained from three experiments; a total of 512 spheroids were analyzed. Data are presented as the means \pm standard error of the mean. ${ }^{*} \mathrm{P}<0.05$. AQP2, aquaporin 2; E2, $17 \beta$-estradiol; siRNA, small interfering RNA.

in uterine receptivity and affect embryo attachment, which is the initial step of implantation. This hypothesis was tested in vitro by knocking down AQP2 via siRNA in the JAr-Ishikawa cell attachment assay (23-25). In the present study, the Ishikawa cell line was selected as a model for receptive endometrium as these cells possess well-reported receptivity and express various implantation-associated molecules (27), as well as estrogen and progesterone receptors (28). JAr is a trophoblastic choriocarcinoma cell line, which exhibits cytotrophoblastic characteristics (29) and is able to attach to Ishikawa cells in vitro (30). The present study demonstrated that knockdown of AQP2 by siRNA in Ishikawa cells significantly suppressed embryo attachment; this finding further supported the hypothesis that AQP2 serves a key role in uterine receptivity and embryo implantation. Our previous study reported an association between AQP2 and E2 in the human endometrium (15). Additionally, the present study revealed the regulatory effects of E2 on AQP2. Specifically, E2 had an effect on the expression of AQP2, but not on JAr attachment to Ishikawa cells compared with the control group. Therefore, these results suggested that E2 may have an indirect effect on endometrial receptivity. These findings in healthy women were in accordance with those of a recent report, which demonstrated that impaired endometrial receptivity in patients following controlled ovarian stimulation is associated with reduced AQP2 expression (31).

Estrogens serve crucial roles in endometrial cellular functions, including cell homeostasis, proliferation, differentiation and vascularization $(32,33)$. ERs are expressed in endometrial glands and the stroma, and genomic experiments have 
demonstrated that estrogens mainly exert their effects via putative ER-EREs in promoter regions (34). A putative ER-ERE was identified in the promoter region of the AQP2 gene (17). Recently, Chai et al (35) compared the effects of high serum E2 levels on endometrial steroid receptors in gonadotropin-stimulated and natural cycles. Analysis revealed that ER expression is significantly reduced in stimulated cycles (35). Therefore, it may be inferred that AQP2 expression and function may be attenuated in the presence of highly elevated serum E2 levels; however, further investigation is required.

Studies have reported significantly lower implantation and pregnancy rates in cycles with high serum E2 concentrations (3-7); however, high serum E2 levels in fresh IVF cycles may not affect implantation and pregnancy rates in subsequent frozen-thawed embryo transfer cycles (36). Therefore, reduced implantation may be due to an adverse endometrial environment resulting from high serum E2 levels and not by the direct action of E2, suggesting that high serum E2 levels may be an indirect cause of reduced implantation (25,31,37-39). The endometrium undergoes a series of precisely regulated alterations under highly elevated serum estrogen levels induced by the application of gonadotrophin in IVF cycles compared with natural cycles $(40,41)$. These changes include impaired development of the endometrial glands (42), advanced stromal development (39), desynchrony between glands and the stroma (36), and early expression of pinopodes (43). The present study revealed that AQP2 expression was decreased under abnormally high E2 levels in human endometrium. This finding suggested a novel molecular mechanism may be involved in reducing endometrial receptivity by elevating serum E2 levels in assisted reproductive technologies, such as IVF. Physiological E2 levels are closer to $10^{-7} \mathrm{M}$; however, at $10^{-5} \mathrm{M}$, E2 may reduce AQP2 expression via ERs (44), which may indicate a different mechanism from that of $10^{-7} \mathrm{ME} 2 \mathrm{used}$ in the present study. Conversely, a previous study proposed that very high serum E2 levels may not be detrimental to the clinical outcomes of IVF within a small sample (45). Further investigation into the underlying mechanism of impaired endometrial receptivity induced by AQP2 is required.

In conclusion, the present study demonstrated that AQP2 expression in human endometrium may be regulated by E2. The in vitro co-culture model using JAr and Ishikawa cells suggested a notable role of AQP2 in uterine receptivity and embryo implantation. Additionally, abnormal AQP2 expression may be a potential cause of impaired uterine receptivity. The results of the present study provided information regarding the causes of decreased uterine receptivity in the context of controlled ovarian hyperstimulation. These findings may provide an important marker (AQP2) for endometrial receptivity in women undergoing IVF. Further studies on the effects of AQP2 expression or polymorphisms on female fertility are required.

\section{Acknowledgements}

This paper has been presented at the 2016 National Symposium on the Advancement of Obstetrics and Gynecology in Integrative Chinese and Western Medicine and 2016 Thesis and Abstracts of the First Jiangsu, Zhejiang, and Zhejiang Integrative Obstetrics and Gynecology Summit Forum.

\section{Funding}

The present study was supported by the National Natural Science Foundation of China (grant nos. 30700273, 81200429 and 81270702), the Natural Science Foundation of Zhejiang Province (grant no. LQ14H040003) and the Fundamental Research Funds for the Central Universities (grant no. 2015FZA7010).

\section{Availability of data and materials}

The datasets used and/or analyzed during the current study are available from the corresponding author on reasonable request.

\section{Authors' contributions}

YZ and RH designed the study. WH, XC and $\mathrm{XH}$ conducted the experiments. $\mathrm{YH}$ and $\mathrm{RH}$ participated in data analysis. $\mathrm{RH}$ and WH wrote the manuscript. All authors read and approved the final version of the manuscript.

\section{Ethics approval and consent to participate}

The present study was approved by the Ethics Committee of the School of Medicine of Zhejiang University. Written informed consent was obtained from each subject prior to participation in this study.

\section{Patient consent for publication}

Written informed consent was obtained from each patient prior to tissue collection and enrolment in the present study.

\section{Competing interests}

The authors declare that they have no competing interests.

\section{References}

1. Adamson GD, Tabangin M, Macaluso M and Mouzon J: The number of babies born globaylly after treatment with the assisted reproductive technologies (ART). Fertil Steril 100: S42, 2013.

2. Paulson RJ, Hatch IE, Lobo RA and Sauer MV: Cumulative conception and live birth rates after oocyte donation: Implications regarding endometrial receptivity. Hum Reprod 12: 835-839, 1997.

3. Absalan F, Ghannadi A and Kazerooni M: Reproductive outcome following thawed embryo transfer in management of ovarian hyperstimulation syndrome. J Reprod Infertil 14: 133-137, 2013.

4. Li MQ and Jin LP: Ovarian stimulation for in vitro fertilization alters the protein profile expression in endometrial secretion. Int J Clin Exp Pathol 6: 1964-1971, 2013.

5. Davar R, Janati S, Mohseni F, Khabazkhoob M and Asgari S: A comparison of the effects of transdermal estradiol and estradiol valerate on endometrial receptivity in frozen-thawed embryo transfer cycles: A randomized clinical trial. J Reprod Infertil 17: 97-103, 2016

6. Kara M, Kutlu T, Sofuoglu K, Devranoglu B and Cetinkaya T: Association between serum estradiol level on the hCG administration day and IVF-ICSI outcome. Iran J Reprod Med 10: 53-58, 2012.

7. Arslan M, Bocca S, Arslan EO, Duran HE, Stadtmauer L and Oehninger S: Cumulative exposure to high estradiol levels during the follicular phase of IVF cycles negatively affects implantation. J Assist Reprod Genet 24: 111-117, 2007.

8. Ullah K, Rahman TU, Pan HT, Guo MX, Dong XY, Liu J, Jin LY, Cheng Y, Ke ZH, Ren J, et al: Serum estradiol levels in controlled ovarian stimulation directly affect the endometrium. J Mol Endocrinol 59: 105-119, 2017. 
9. Dominguez F, Garrido-Gomez T, Lopez JA, Camafeita E, Quinonero A, Pellicer A and Simón C: Proteomic analysis of the human receptive versus non-receptive endometrium using differential in-gel electrophoresis and MALDI-MS unveils stathmin 1 and annexin A2 as differentially regulated. Hum Reprod 24: 2607-2617, 2009.

10. Huang HF, He RH, Sun CC, Zhang Y, Meng QX and Ma YY: Function of aquaporins in female and male reproductive systems. Hum Reprod Update 12: 785-795, 2006.

11. Zhu C, Jiang Z, Bazer FW, Johnson GA, Burghardt RC and Wu G: Aquaporins in the female reproductive system of mammals. Front Biosci (Landmark Ed) 20: 838-871, 2015.

12. Day RE, Kitchen P, Owen DS, Bland C, Marshall L, Conner AC, Bill RM and Conner MT: Human aquaporins: regulators of transcellular water flow. Biochim Biophys Acta 1840: 1492-1506, 2014.

13. Aralla M, Borromeo V, Groppetti D, Secchi C, Cremonesi F and Arrighi S: A collaboration of aquaporins handles water transport in relation to the estrous cycle in the bitch uterus. Theriogenology 72: 310-321, 2009.

14. Skowronski MT, Skowronska A and Nielsen S: Fluctuation of aquaporin 1,5, and 9 expression in the pig oviduct during the estrous cycle and early pregnancy. J Histochem Cytochem 59: 419-427, 2011

15. He RH, Sheng JZ, Luo Q, Jin F, Wang B, Qian YL, Zhou CY, Sheng $X$ and Huang HF: Aquaporin-2 expression in human endometrium correlates with serum ovarian steroid hormones Life Sci 79: 423-429, 2006.

16. Hildenbrand A, Lalitkumar L, Nielsen S, Gemzell-Danielsson K and Stavreus-Evers A: Expression of aquaporin 2 in human endometrium. Fertil Steril 86: 1452-1458, 2006.

17. Zou LB, Zhang RJ, Tan YJ, Ding GL, Shi S, Zhang D, He RH, Liu AX, Wang TT, Leung PC, et al: Identification of estrogen response element in the aquaporin-2 gene that mediates estrogen-induced cell migration and invasion in human endometrial carcinoma. J Clin Endocrinol Metab 96: E1399-E1408, 2011

18. Noyes RW, Hertig AT and Rock J: Dating the endometrial biopsy. Am J Obstet Gynecol 122: 262-263, 1975.

19. Horne AW, King AE, Shaw E, McDonald SE, Williams AR Saunders PT and Critchley HO: Attenuated sex steroid receptor expression in fallopian tube of women with ectopic pregnancy. J Clin Endocrinol Metab 94: 5146-5154, 2009.

20. Fushimi K, Uchida S, Hara Y, Hirata Y, Marumo F and Sasaki S Cloning and expression of apical membrane water channel of rat kidney collecting tubule. Nature 361: 549-552, 1993

21. Schefe JH, Lehmann KE, Buschmann IR, Unger $T$ and Funke-Kaiser H: Quantitative real-time RT-PCR data analysis: Current concepts and the novel 'gene expression's CT difference' formula. J Mol Med (Berl) 84: 901-910, 2006.

22. Livak KJ and Schmittgen TD: Analysis of relative gene expression data using real-time quantitative PCR and the 2(-Delta Delta C(T)) method. Methods 25: 402-408, 2001

23. Hohn HP, Linke M and Denker HW: Adhesion of trophoblast to uterine epithelium as related to the state of trophoblast differentiation: In vitro studies using cell lines. Mol Reprod Dev 57: 135-145, 2000.

24. Uchida H, Maruyama T, Ohta K, Ono M, Arase T, Kagami M, Oda H, Kajitani T, Asada H and Yoshimura Y: Histone deacetylase inhibitor-induced glycodelin enhances the initial step of implantation. Hum Reprod 22: 2615-2622, 2007.

25. Liu Y, Kodithuwakku SP, Ng PY, Chai J, Ng EH, Yeung WS, Ho PC and Lee KF: Excessive ovarian stimulation up-regulates the Wnt-signaling molecule DKK1 in human endometrium and may affect implantation: An in vitro co-culture study. Hum Reprod 25: 479-490, 2010.

26. Marsh EE, Shaw ND, Klingman KM, Tiamfook-Morgan TO, Yialamas MA, Sluss PM and Hall JE: Estrogen levels are higher across the menstrual cycle in African-American women compared with Caucasian women. J Clin Endocrinol Metab 96: 3199-3206, 2011

27. Hannan NJ, Paiva P, Dimitriadis E and Salamonsen LA: Models for study of human embryo implantation: Choice of cell lines? Biol Reprod 82: 235-245, 2010.
28. Nishida M: The Ishikawa cells from birth to the present. Hum Cell 15: 104-117, 2002.

29. Apps R, Murphy SP, Fernando R, Gardner L, Ahad T and Moffett A: Human leucocyte antigen (HLA) expression of primary trophoblast cells and placental cell lines, determined using single antigen beads to characterize allotype specificities of anti-HLA antibodies. Immunology 127: 26-39, 2009.

30. Heneweer C, Schmidt M, Denker HW and Thie M: Molecular mechanisms in uterine epithelium during trophoblast binding: The role of small GTPase RhoA in human uterine Ishikawa cells. J Exp Clin Assist Reprod 2: 4, 2005.

31. Zhang D, Xu G, Zhang R, Zhu Y, Gao H, Zhou C, Sheng J and Huang $\mathrm{H}$ : Decreased expression of aquaporin 2 is associated with impaired endometrial receptivity in controlled ovarian stimulation. Reprod Fertil Dev 28: 499-506, 2016.

32. Smith SK: Regulation of angiogenesis in the endometrium. Trends Endocrinol Metab 12: 147-151, 2001.

33. Gupta SC, Kim JH, Prasad S and Aggarwal BB: Regulation of survival, proliferation, invasion, angiogenesis, and metastasis of tumor cells through modulation of inflammatory pathways by nutraceuticals. Cancer Metastasis Rev 29: 405-434, 2010.

34. Klinge CM, Jernigan SC, Mattingly KA, Risinger KE and Zhang J: Estrogen response element-dependent regulation of transcriptional activation of estrogen receptors alpha and beta by coactivators and corepressors. J Mol Endocrinol 33: 387-410, 2004.

35. Chai J, Lee KF, Ng EH, Yeung WS and Ho PC: Ovarian stimulation modulates steroid receptor expression and spheroid attachment in peri-implantation endometria: Studies on natural and stimulated cycles. Fertil Steril 96: 764-768, 2011.

36. Yu Ng EH, Yeung WS, Yee Lan Lau E, So WW and Ho PC: High serum oestradiol concentrations in fresh IVF cycles do not impair implantation and pregnancy rates in subsequent frozen-thawed embryo transfer cycles. Hum Reprod 15: 250-255, 2000.

37. Forman R, Fries N, Testart J, Belaisch-Allart J, Hazout A and Frydman R: Evidence for an adverse effect of elevated serum estradiol concentrations on embryo implantation. Fertil Steril 49: 118-122, 1988.

38. Fossum GT, Davidson A and Paulson RJ: Ovarian hyperstimulation inhibits embryo implantation in the mouse. J In Vitro Fert Embryo Transf 6: 7-10, 1989.

39. Noci I, Borri P, Coccia ME, Criscuoli L, Scarselli G, Messeri G, Paglierani M, Moncini D and Taddei G: Hormonal patterns, steroid receptors and morphological pictures of endometrium in hyperstimulated IVF cycles. Eur J Obstet Gynecol Reprod Biol 75: 215-220, 1997.

40. Kalem Z, Kalem MN and Gurgan T: Methods for endometrial preparation in frozen-thawed embryo transfer cycles. J Turk Ger Gynecol Assoc 17: 168-172, 2016.

41. Valbuena D, Jasper M, Remohi J, Pellicer A and Simon C: Ovarian stimulation and endometrial receptivity. Hum Reprod (2 Suppl 14): S107-S111, 1999

42. Xu LZ, Gao MZ, Yao LH, Liang AJ, Zhao XM and Sun ZG: Effect of high ovarian response on the expression of endocrine gland-derived vascular endothelial growth factor (EG-VEGF) in peri-implantation endometrium in IVF women. Int J Clin Exp Pathol 8: 8902-8911, 2015.

43. Rashidi B, Soleimani Rad JI, Roshangar L and Alizadeh Miran R: Evaluation of pinopodes expression on the mouse endometrium immediately before implantation by treatment with $\mathrm{HMG} / \mathrm{HCG}$ and sildenafil citrate administration. Iran J Basic Med Sci 15: 1091-1096, 2012.

44. Cheema MU, Irsik DL, Wang Y, Miller-Little W, Hyndman KA, Marks ES, Frøkiær J, Boesen EI and Norregaard R: Estradiol regulates AQP2 expression in the collecting duct: A novel inhibitory role for estrogen receptor alpha. Am J Physiol Renal Physiol 309: F305-F317, 2015.

45. Chenette PE, Sauer MV and Paulson RJ: Very high serum estradiol levels are not detrimental to clinical outcome of in vitro fertilization. Fertil Steril 54: 858-863, 1990 